Supporting Information for the Paper Entitled:

\title{
A Nitridoniobium(V) Reagent that Effects Acid Chloride to Organic Nitrile Conversion: Synthesis via Heterodinuclear (Nb/Mo) Dinitrogen Cleavage, Mechanistic Insights, and Recycling
}

\author{
Joshua S. Figueroa, Nicholas A. Piro, Christopher R. Clough and Christopher C. Cummins* \\ Department of Chemistry, Room 2-227, Massachusetts Institute of Technology \\ 77 Massachusetts Avenue, Cambridge, MA 02139-4307 \\ Email: ccummins@mit.edu
}

\section{CONTENTS}

S1. ${ }^{15} \mathrm{~N}$ NMR Spectra for ${ }^{15} \mathrm{~N}$-Labeled Nitrido Niobium and Molybenum Complexes and ${ }^{15} \mathrm{~N}$-Labeled Organonitriles .............................................. S

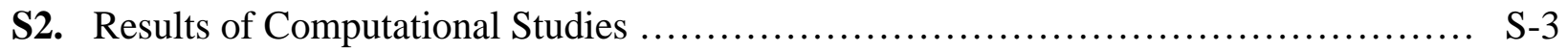

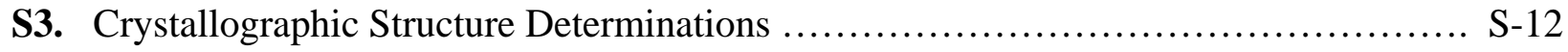




\section{S1. ${ }^{15} \mathrm{~N}$ NMR Spectra for ${ }^{15} \mathrm{~N}$-Labeled Organonitriles and ${ }^{15} \mathrm{~N}$-Labeled Nitrido Niobium and Molybenum Complexes.}

Solution ${ }^{15} \mathrm{~N}$ NMR spectra were recorded on a Bruker Advance-600 spectrometer operating at a resonance frequency of $60.84 \mathrm{MHz} .{ }^{15} \mathrm{~N}$ NMR chemical shifts are reported referenced to external formamide- ${ }^{15} \mathrm{~N}\left(\mathrm{H}_{2}{ }^{15} \mathrm{NC}(\mathrm{O}) \mathrm{H}, 55 / 45 \mathrm{v} / \mathrm{v}\right.$ in DMSO); $113.0 \mathrm{ppm}$ relative to liquid $\mathrm{NH}_{3}(0.0 \mathrm{ppm}) .{ }^{1}$

$\boldsymbol{A}$
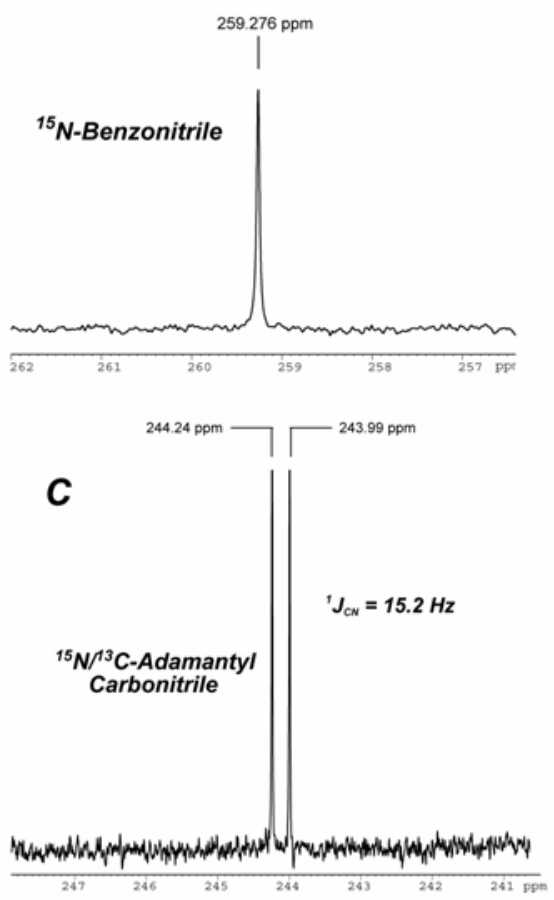

B

${ }^{15} \mathrm{~N}$-Pivalonitrile

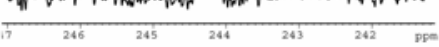

D
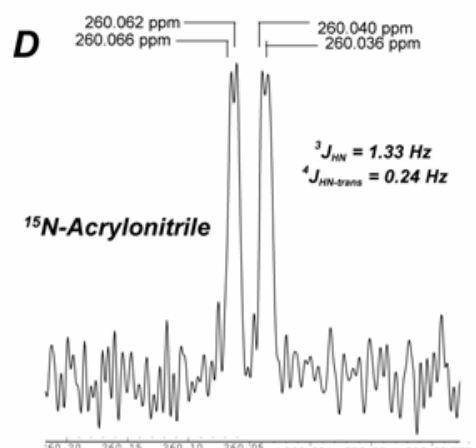

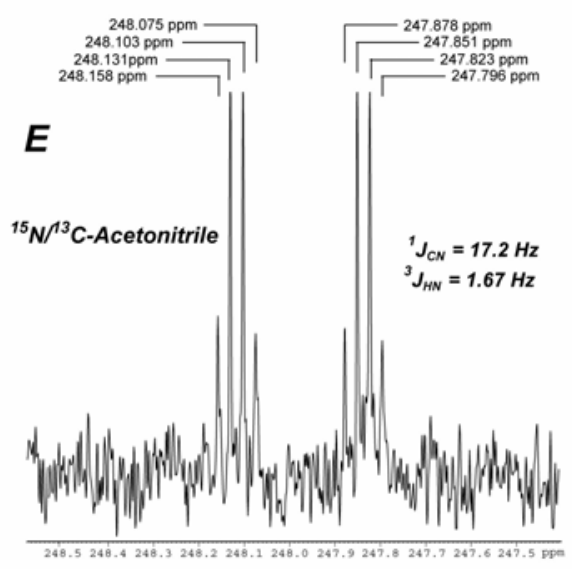

Figure S1-1. Solution ${ }^{15} \mathrm{~N}$ NMR Spectra for ${ }^{15} \mathrm{~N}$-Labeled Organonitriles prepared in this study. All spectra were acquired in THF- $d_{8}$ at $20.1{ }^{\circ} \mathrm{C}$ on an instrument operating at $60.84 \mathrm{MHz}\left({ }^{1} \mathrm{H}=600.2 \mathrm{MHz}\right)$. 


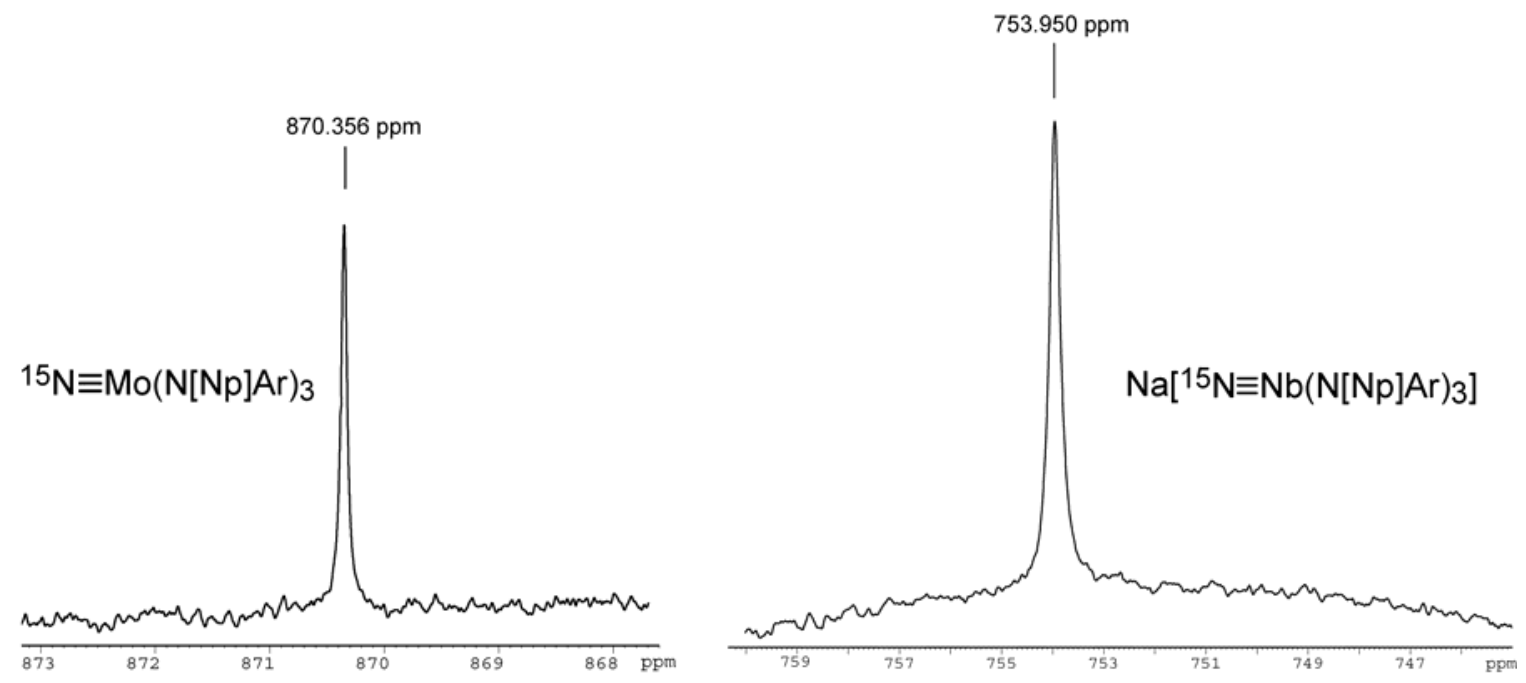

Figure S1-2. Solution ${ }^{15} \mathrm{~N}$ NMR Spectra ${ }^{15} \mathrm{~N}$-Labeled $\mathrm{N} \equiv \mathrm{Mo}(\mathrm{N}[\mathrm{Np}] \mathrm{Ar})_{3}$ and $\mathrm{Na}\left[{ }^{15} \mathrm{~N} \equiv \mathrm{Nb}(\mathrm{N}[\mathrm{Np}] \mathrm{Ar})_{3}\right]$. Both spectra were acquired in THF- $d_{8}$ at $20.1^{\circ} \mathrm{C}$ on an instrument operating at $60.84 \mathrm{MHz}\left({ }^{1} \mathrm{H}=600.2 \mathrm{MHz}\right)$.

\section{S2. Results of Computational Studies}

All DFT calculations were carried out utilizing the Amsterdam Density Functional (ADF) program suite, ${ }^{2,3}$ version 2004.01. ${ }^{4}$ The all-electron, Slater-type orbital (STO) basis sets employed were of triple$\zeta$ quality augmented with two polarization functions and incorporated relativistic effects using the zeroorder regular approximation ${ }^{5,6}$ (ADF basis ZORA/TZ2P). The local exchange-correlation potential of Vosko et al. ${ }^{7}$ (VWN) was augmented self-consistently with gradient-corrected functionals for electron exchange according to Becke, ${ }^{8}$ and electron correlation according to Perdew. ${ }^{9,10}$ This nonlocal density functional is termed BP86 in the literature and has been shown to give excellent results for the geometries and energetics of transition metal systems. ${ }^{11}$ Crystallographically determined atomic coordinates were used as input where appropriate. Each optimized structure was subjected to a harmonic frequency calculation to ensure representation as local minima on the potential energy surface. All enthalpic values reported were corrected for zero-point and internal energy considerations (SATP; $298.15 \mathrm{~K}, 1.0 \mathrm{~atm})$.

\section{S2.1 Representative Input File: Input for the Niobacyclobutene Complex Bent-(MeC(O)N)Nb( $\left.\mathrm{NH}_{2}\right)_{3}(4 \mathrm{~m}-\mathrm{B})$.}

\$ADFBIN/adf - n8 $<$ eor

TITLE Bent $\mathrm{Nb}$ Acylimido Geometry opt

MAXMEMORYUSAGE 768

RELATIVISTIC ZORA

RESTRICTED

CHARGE $\odot$ 
$X C$

LDA VWN

GGA Becke Perdew

END

SYMMETRY NOSYM

ATOMS

C $\quad 2.679198$

$0.301641 \quad 3.304082$

$\begin{array}{llll}\text { C } & 1.669522 & 0.005762 & 2.230428\end{array}$

$\begin{array}{llll}0 & 2.136195 & -0.181013 & 1.023294\end{array}$

$\begin{array}{llll}\mathrm{N} & 0.344951 & -0.095053 & 2.406346\end{array}$

$\begin{array}{llll}\mathrm{Nb} & 0.427633 & -0.036236 & 0.204848\end{array}$

$\begin{array}{llll}N & -1.154020 & -0.865456 & -0.307630\end{array}$

$\mathrm{N} \quad 0.511150 \quad 1.867362 \quad-0.456655$

$\mathrm{N} \quad 1.774086 \quad-1.105353 \quad-1.070810$

$\begin{array}{llll}\mathrm{H} & 2.196648 & 0.453328 & 4.276157\end{array}$

$\begin{array}{llll}\text { H } & 3.383913 & -0.542676 & 3.370565\end{array}$

$\begin{array}{llll}\mathrm{H} & 3.266327 & 1.192408 & 3.033188\end{array}$

$\begin{array}{llll}\mathrm{H} & 2.652409 & -1.516936 & -0.752342\end{array}$

$\mathrm{H} \quad 1.437185 \quad-1.522946 \quad-1.937856$

$\begin{array}{llll}\mathrm{H} & -0.019272 & 2.612759 & 0.140610\end{array}$

$\mathrm{H} \quad 0.705248 \quad 2.248159 \quad-1.331828$

$\begin{array}{llll}H & -1.962075 & -0.327269 & -0.620721\end{array}$

$\begin{array}{llll}\mathrm{H} & -1.417531 & -1.840588 & -0.160338\end{array}$

END

GEOMETRY

GO

END

BASIS

type TZ2P

core none

END

END INPUT

Eor 
S2.2 Optimized Structures and Coordinates for Models $4 \mathrm{~m}$ and $\mathrm{MeC} \equiv \mathrm{N}$ (RESULTS).

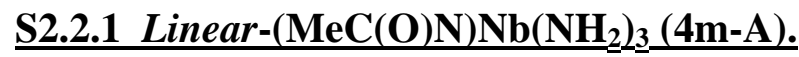

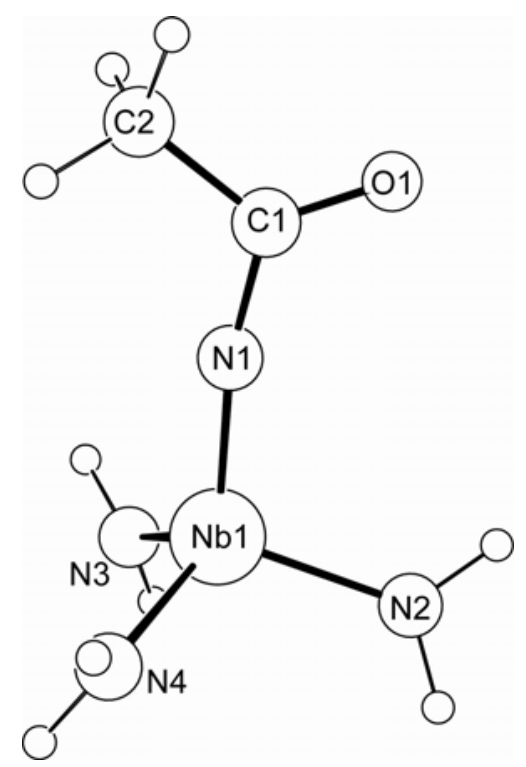

Figure S2.1. Optimized Geometry of Linear-(MeC(O)N)Nb(N[Np]Ar) $\left.)_{3}\right](\mathbf{4 m - A})$.

\section{Linear- $(\mathrm{MeC}(\mathrm{O}) \mathrm{N}) \mathrm{Nb}\left(\mathrm{NH}_{2} 2\right)_{3}, 4 \mathrm{~m}-\mathrm{A}$ (Cartesian).}

\begin{tabular}{lrrr}
\hline $\mathrm{H}$ & -0.331232 & -2.157760 & -3.164296 \\
$\mathrm{~N}$ & -0.549609 & -1.449980 & -2.461372 \\
$\mathrm{Nb}$ & 0.331642 & -1.361253 & -0.668976 \\
$\mathrm{~N}$ & -0.693137 & -2.475851 & 0.641627 \\
$\mathrm{H}$ & -0.771433 & -2.298683 & 1.642429 \\
$\mathrm{H}$ & -1.002295 & -0.659269 & -2.919791 \\
$\mathrm{~N}$ & 2.224452 & -1.986434 & -0.753649 \\
$\mathrm{H}$ & 2.514392 & -2.960932 & -0.671443 \\
$\mathrm{H}$ & 3.035544 & -1.377679 & -0.652569 \\
$\mathrm{~N}$ & 0.419416 & 0.350056 & -0.123409 \\
$\mathrm{C}$ & 0.609289 & 1.609262 & 0.424149 \\
$\mathrm{C}$ & -0.663415 & 2.414348 & 0.648112 \\
$\mathrm{H}$ & -0.456658 & 3.240688 & 1.336470 \\
$\mathrm{O}$ & 1.720317 & 2.055177 & 0.688622 \\
$\mathrm{H}$ & -1.355677 & -3.204617 & 0.376550 \\
$\mathrm{H}$ & -0.993890 & 2.830394 & -0.314766 \\
$\mathrm{H}$ & -1.473900 & 1.782664 & 1.031534
\end{tabular}




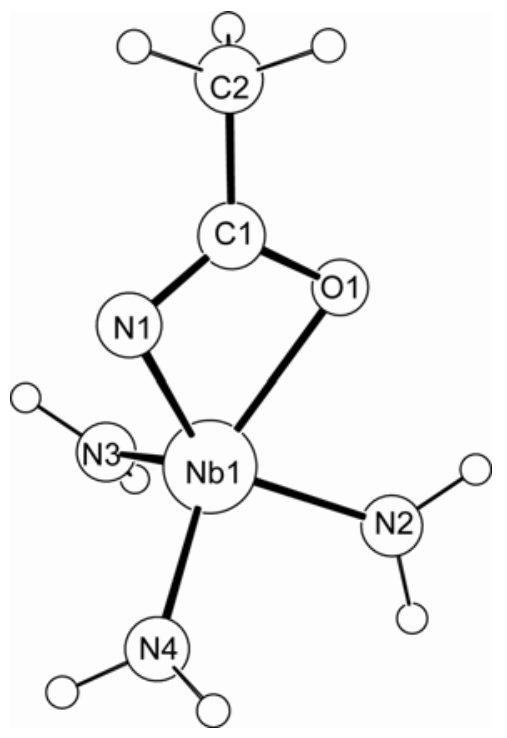

Figure S2.2. Optimized Geometry of Bent--(MeC(O)N)Nb($\left(\mathrm{NH}_{2}\right)_{3}(\mathbf{4 m - B})$.

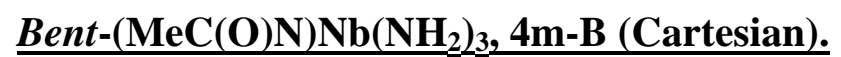

\begin{tabular}{lrrr}
\hline $\mathrm{C}$ & 2.550910 & 0.306347 & 3.506100 \\
$\mathrm{C}$ & 1.746598 & -0.008161 & 2.276118 \\
$\mathrm{O}$ & 2.391229 & -0.409545 & 1.194678 \\
$\mathrm{~N}$ & 0.437613 & 0.115061 & 2.161352 \\
$\mathrm{Nb}$ & 0.475178 & -0.021996 & 0.183281 \\
$\mathrm{~N}$ & -1.238017 & -0.941602 & -0.265068 \\
$\mathrm{~N}$ & 0.258463 & 1.830282 & -0.459349 \\
$\mathrm{~N}$ & 1.544632 & -1.030047 & -1.164189 \\
$\mathrm{H}$ & 1.895109 & 0.520979 & 4.355353 \\
$\mathrm{H}$ & 3.204482 & -0.546358 & 3.736962 \\
$\mathrm{H}$ & 3.203234 & 1.168937 & 3.310195 \\
$\mathrm{H}$ & 2.472710 & -1.409910 & -0.982200 \\
$\mathrm{H}$ & 1.179292 & -1.332644 & -2.066468 \\
$\mathrm{H}$ & 0.030226 & 2.596965 & 0.173649 \\
$\mathrm{H}$ & 0.533332 & 2.190012 & -1.372789 \\
$\mathrm{H}$ & -2.130157 & -0.453589 & -0.328504 \\
$\mathrm{H}$ & -1.387515 & -1.946667 & -0.342501
\end{tabular}




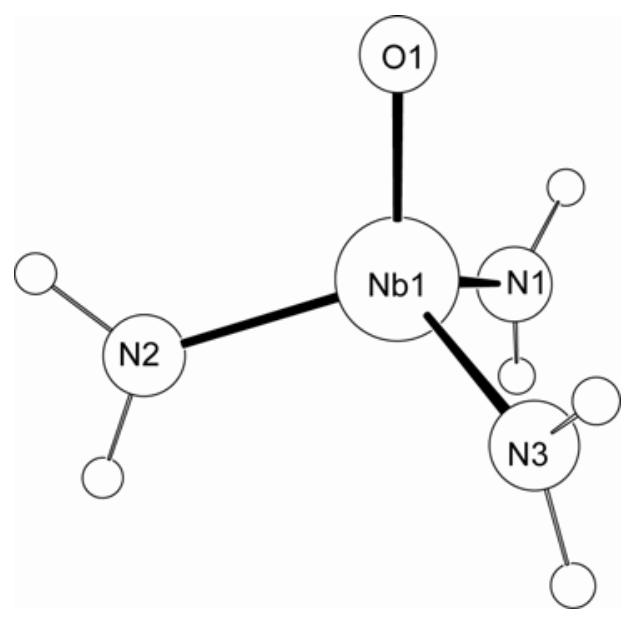

Figure S2.3. Optimized Geometry of $\mathrm{O} \equiv \mathrm{Nb}\left(\mathrm{NH}_{2}\right)_{3}(\mathbf{4 m - O})$.

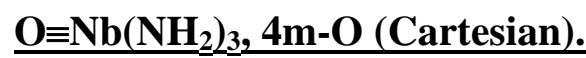

\begin{tabular}{lrrr}
\hline $\mathrm{H}$ & 0.801209 & -1.513442 & -2.280293 \\
$\mathrm{~N}$ & 0.525735 & -1.581241 & -1.301191 \\
$\mathrm{Nb}$ & 0.151488 & -0.063570 & -0.045727 \\
$\mathrm{O}$ & 0.068733 & -0.705057 & 1.544917 \\
$\mathrm{H}$ & 0.549673 & -2.557606 & -1.009622 \\
$\mathrm{~N}$ & -1.621440 & 0.785884 & -0.438940 \\
$\mathrm{H}$ & -2.376352 & 0.830191 & 0.244073 \\
$\mathrm{H}$ & -1.956014 & 1.141278 & -1.333512 \\
$\mathrm{~N}$ & 1.629271 & 1.290204 & -0.118913 \\
$\mathrm{H}$ & 2.252258 & 1.484804 & 0.664331 \\
$\mathrm{H}$ & 1.824480 & 1.945369 & -0.875041
\end{tabular}

\section{$\underline{\text { S2.2.4 } \mathrm{MeC} \equiv \mathrm{N}}$.}

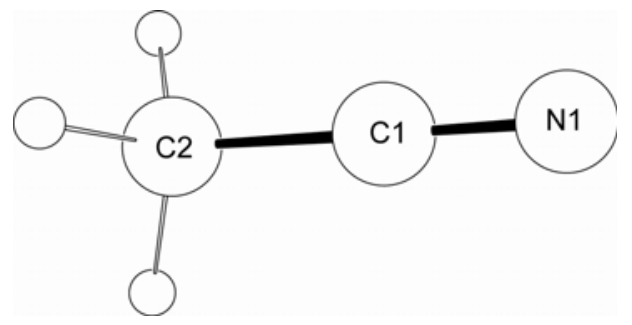

Figure S2.4. Optimized Geometry of $\mathrm{MeC} \equiv \mathrm{N}$.

\section{$\underline{\mathrm{MeC}} \equiv \mathbf{N}$ (Cartesian)}

\begin{tabular}{lrrr}
\hline $\mathrm{N}$ & 0.002316 & $0.00 \odot \odot 12$ & -0.030671 \\
$\mathrm{C}$ & -0.004369 & $-0.000 \odot 14$ & 1.130404 \\
$\mathrm{C}$ & 0.001042 & $-0.00 \odot \odot \odot 8$ & 2.587067
\end{tabular}




$\begin{array}{rrrr}H & 1.032234 & 0.000036 & 2.962332 \\ H & -0.512396 & 0.891895 & 2.967775 \\ H & -0.512416 & -0.891828 & 2.967941\end{array}$

\section{S2.3. Comparison of Metrical Parameters Between Experimental and Calculated Structures.}

Table S2.1. Comparison of Metrical Parameters Between Experimental and Calculated Structures for Complexes 1c-NC(O)t-Bu and $\mathbf{4 m - A}$ (See Figure S2.1 for numbering scheme).

\begin{tabular}{lccc} 
Parameter & Crystallographic & Calculated & \% Difference \\
\hline Nb1-N1 & & & \\
Nb1-N2 & $1.818(6)$ & 1.798 & 1.10 \\
Nb1-N3 & $2.005(6)$ & 1.995 & 0.49 \\
Nb1-N4 & $2.012(6)$ & 2.003 & 0.44 \\
N1-C1 & $2.027(6)$ & 1.999 & 1.38 \\
C1-O1 & $1.389(10)$ & 1.386 & 0.21 \\
C1-C2 & $1.234(10)$ & 1.226 & 0.65 \\
Nb1-N1-C1 & $1.549(11)$ & 1.523 & 1.67 \\
N1-C1-O1 & $167.7(6)$ & 172.3 & 2.70 \\
\hline
\end{tabular}

Table S2.2. Comparison of Metrical Parameters Between Experimental and Calculated Structures for Complexes 1a-O and $\mathbf{4 m - O}$ (See Figure S2.3 for numbering scheme).

\begin{tabular}{llcc} 
Parameter & Crystallographic & Calculated & \% Difference \\
\hline Nb1-O1 & & & \\
Nb1-N1 & $1.697(3)$ & 1.716 & 1.12 \\
Nb1-N2 & $2.001(3)$ & 2.006 & 0.24 \\
Nb1-N3 & $2.012(3)$ & 2.006 & 0.30 \\
O1-Nb1-N1 & $2.011(3)$ & 2.006 & 0.30 \\
O1-Nb1-N2 & $103.87(8)$ & 108.15 & 4.12 \\
N1-Nb1-N2 & $102.92(8)$ & 108.07 & 5.00 \\
\hline
\end{tabular}

S2.4. Calculated Harmonic Frequencies and Relative Absorption Intensities for Models 4m and $\mathrm{MeC} \equiv \mathrm{N}$.

S2.4.1 Calculated Harmonic Frequencies and Relative Absorption Intensities for Linear-( $\mathrm{MeC}(\mathrm{O}) \mathrm{N}) \mathrm{Nb}\left(\mathrm{NH}_{2}\right)_{3}(4 \mathrm{~m}-\mathrm{A})$.

$$
\begin{gathered}
\begin{array}{c}
\text { Frequency } \\
\text { cm-1 }
\end{array} \\
------ \\
13.321895 \\
48.258533 \\
56.920187
\end{gathered}
$$

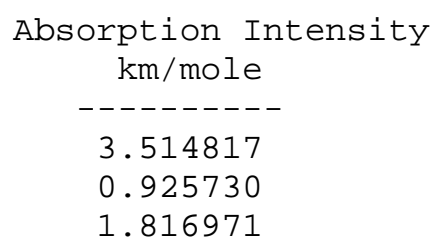




\begin{tabular}{rr}
65.746326 & 0.281960 \\
136.381469 & 4.546089 \\
161.771099 & 4.547377 \\
168.826250 & 1.948426 \\
183.254905 & 2.928625 \\
198.434270 & 0.870054 \\
255.848762 & 7.708407 \\
281.363896 & 11.300640 \\
336.966213 & 11.891935 \\
344.803003 & 8.325164 \\
431.121046 & $10 \odot .293660$ \\
444.622499 & 79.172869 \\
465.455979 & 137.638258 \\
522.362117 & 6.914900 \\
535.457261 & 11.441852 \\
554.836540 & 5.778509 \\
565.468067 & 1.022565 \\
573.935302 & 5.780613 \\
609.689613 & 61.948050 \\
642.946580 & 58.527653 \\
649.535213 & 149.701308 \\
654.129467 & 144.653706 \\
935.912618 & 55.592135 \\
1007.813359 & 3.992983 \\
1053.407347 & 21.558718 \\
1276.783243 & 936.631069 \\
1326.895238 & 75.301713 \\
1418.493946 & 12.960182 \\
1425.745301 & 9.794888 \\
1487.861122 & 46.011636 \\
1489.544101 & 48.009731 \\
1493.343970 & 9.112741 \\
1644.341390 & 340.777347 \\
\hline 2966.667565 & 4.114777 \\
3035.778408 & 6.472212 \\
3070.743648 & 8.999215 \\
3427.245167 & 56.524961 \\
3434.416070 & 60.225855 \\
3438.151550 & 32.318301 \\
3520.400461 & 18.272468 \\
3531.132296 & 17.739395 \\
3531.760035 & 22.567133
\end{tabular}

Note: $v(C=0)$

S2.4.2 Calculated Harmonic Frequencies and Relative Absorption Intensities for Bent-(MeC(O)N)Nb( $\left.\mathrm{NH}_{2}\right)_{3}(4 \mathrm{~m}-\mathrm{B})$.

$\begin{gathered}\text { Frequency } \\ \mathrm{cm}-1\end{gathered}$
------
41.622639
54.884897
75.099885
88.859349
156.385786
163.924280
198.207149
205.301771
242.126449
284.880521
365.763534
386.537062

$\begin{gathered}\text { Absorption Intensity } \\ \mathrm{km} / \mathrm{mole}\end{gathered}$
-3.081684
2.265289
0.915496
1.956526
1.362507
4.567468
1.234943
2.984755
4.663559
5.068402
30.377957
10.392736




\begin{tabular}{rr}
398.291747 & 134.861709 \\
443.448794 & 46.222570 \\
454.045304 & 106.104045 \\
505.774024 & 6.147412 \\
525.750337 & 9.935929 \\
538.128256 & 2.725148 \\
557.217264 & 3.183264 \\
580.041616 & 2.241513 \\
605.380444 & 50.049083 \\
643.249454 & 95.331302 \\
652.190393 & 165.246927 \\
677.031754 & 99.773233 \\
741.017935 & 181.596088 \\
906.698845 & 45.119673 \\
962.790557 & 17.101116 \\
1015.853892 & 1.316307 \\
1291.221822 & 97.956546 \\
1336.992543 & 32.060200 \\
1397.285883 & 159.112057 \\
1423.098825 & 8.968772 \\
1459.050094 & 137.109009 \\
1474.341942 & 129.579377 \\
1479.161643 & 44.497125 \\
$\mathbf{1 4 8 5 . 3 0 1 9 9 9}$ & 12.217863 \\
\hline 2967.831646 & 4.360678 \\
\hline 3029.292847 & 3.594330 \\
3076.065870 & 3.884408 \\
3428.506701 & 30.643153 \\
3434.009991 & 57.062472 \\
3442.644068 & 44.491109 \\
3534.961918 & 24.579476 \\
3539.909590 & 17.579512 \\
3541.612882 & 15.398007
\end{tabular}

Note: $v(C=0)$ Not Present

\section{S2.4.3 Calculated Harmonic Frequencies and Relative Absorption Intensities for $\mathrm{O} \equiv \mathrm{Nb}\left(\mathrm{NH}_{2}\right)_{3}(4 \mathrm{~m}-\mathrm{O})$.}

\begin{tabular}{|c|c|}
\hline $\begin{array}{l}\text { Frequency } \\
\text { cm-1 }\end{array}$ & $\begin{array}{l}\text { Absorption Intensity } \\
\mathrm{km} / \mathrm{mole}\end{array}$ \\
\hline - - - - - - - - & ------- \\
\hline 105.542101 & 0.978785 \\
\hline 172.273513 & 4.326885 \\
\hline 175.183505 & 4.262622 \\
\hline 183.987515 & 3.156554 \\
\hline 197.241479 & 0.345212 \\
\hline 199.671304 & 0.193033 \\
\hline 350.882586 & 5.616618 \\
\hline 356.918738 & 6.319447 \\
\hline 397.841705 & 6.708070 \\
\hline 417.380709 & 209.668269 \\
\hline 419.612835 & 215.570365 \\
\hline 530.824126 & 1.954572 \\
\hline 536.526603 & 1.793155 \\
\hline 555.263814 & 7.329121 \\
\hline 638.381821 & 32.718984 \\
\hline 639.984211 & 164.026074 \\
\hline 641.898122 & 158.367753 \\
\hline 976.987458 & 153.034944 \\
\hline 1492.647895 & 41.890493 \\
\hline
\end{tabular}




$\begin{array}{lr}1494.005992 & 42.923126 \\ 1498.731597 & 5.923611 \\ 3438.753779 & 59.018328 \\ 3440.710337 & 50.678320 \\ 3444.549869 & 19.743550 \\ 3533.793088 & 20.177253 \\ 3536.166185 & 21.101132 \\ 3538.325032 & 19.963563\end{array}$

S2.4.4 Calculated Harmonic Frequencies and Relative Absorption Intensities for $\mathrm{MeC} \equiv \mathbf{N}$.

\begin{tabular}{|c|c|c|}
\hline $\begin{array}{l}\text { Frequency } \\
\mathrm{cm}-1\end{array}$ & $\begin{array}{c}\text { Absorption Inte } \\
\mathrm{km} / \mathrm{mole}\end{array}$ & isity \\
\hline - - - - - - - - & $--\ldots$ & \\
\hline 365.634317 & 0.183168 & \\
\hline 366.003448 & $\odot .206547$ & \\
\hline 913.125974 & $\odot .42821 \odot$ & \\
\hline 1021.604920 & 1.831881 & \\
\hline 1022.321310 & 1.795703 & \\
\hline 1361.166449 & 1.676733 & \\
\hline 1425.772704 & 10.926604 & \\
\hline 1425.844314 & 10.891918 & \\
\hline 2275.766186 & 11.241956 & Note: $v(C \equiv N)$ \\
\hline 2976.737346 & 1.674010 & \\
\hline 3048.872931 & $\odot .303368$ & \\
\hline 3048.942853 & $\odot .276169$ & \\
\hline
\end{tabular}

S2.5 Relative Thermodynamic Parameters, Zero Point Energy and Internal Energy Corrections (kcal/mol).

\begin{tabular}{lcccc} 
Model & $\begin{array}{c}\text { Total Bonding } \\
\text { Energy (TBE) }\end{array}$ & $\begin{array}{c}\text { Zero Point } \\
\text { Energy }\end{array}$ & $\begin{array}{c}\text { Internal Energy } \\
\text { Correction* }\end{array}$ & Corrected TBE** \\
\hline 4m-A & -2353.27 & 76.36 & 84.181 & -2269.08 \\
4m-B & -2351.04 & 76.92 & 84.882 & -2266.15 \\
4m-O & -1521.62 & 47.05 & 52.791 & -1468.83 \\
$\mathbf{M e C} \equiv \mathbf{N}$ & -837.75 & 27.52 & 29.820 & -807.93
\end{tabular}

* Internal energy corrected to Standard Ambient Temperature and Pressure (SATP; 298.15 K, 1.0 ATM)

**Corrected TBE = TBE + (Internal Energy Correction), where the Internal Energy Correction includes Zero-Point Energy considerations.

\section{S3. Crystallographic Structure Determinations}

\section{S3.1 Crystallographic Information for complexes $\mathrm{Nb}(\mathrm{I})(\mathrm{N}[\mathrm{Np}] \mathrm{Ar})_{3} \quad(1 \mathrm{a}-\mathrm{I})$ and $\mathrm{Nb}(\mathrm{OTf})(\mathrm{N}[\mathrm{Np}] \mathrm{Ar})_{3}\left(\mathrm{aa}-\mathrm{OTf}, \mathrm{OTf}=\mathrm{OSO}_{2} \mathrm{CF}_{3}\right)$}

The X-ray crystallographic data collections were carried out on a Siemens Platform three-circle diffractometer mounted with an APEX-CCD detector and outfitted with a low-temperature, nitrogenstream aperture. The structures were solved using direct methods, in conjunction with standard difference Fourier techniques and refined by full-matrix least-squares procedures. ORTEP depictions of 
complexes 1a-I and 1a-OTf can be found in Figures S3.1 and S3.2, respectively. Summaries of crystallographic data for all complexes are given in Tables S3.1 and S3.2. Experimental details for the crystallographic structure determinations for complexes $\mathbf{2 b}-\mathrm{H}, \mathrm{Na}(\mathrm{THF})_{3}[\mathbf{1 a}-\mathrm{N}], \mathbf{2} \mathbf{b}-\mathrm{N}$ and $\mathbf{1 c}-\mathrm{NC}(\mathrm{O}) t-$ $\mathrm{Bu}$ can be found in the Main Text. The systematic absences in the diffraction data are uniquely consistent with the assigned monoclinic space groups of $\mathrm{P} 2{ }_{1} / \mathrm{n}$ for complex 1a-I. No symmetry higher than triclinic was indicated in the diffraction data for complex 1a-OTf. These choices led to chemically sensible and computationally stable refinements. An empirical absorption correction (SADABS) was applied to the diffraction data for both structures. All non-hydrogen atoms were refined anisotropically. Hydrogen atoms were treated as idealized contributions and refined isotropically. All software used for diffraction data processing and crystal-structure solution and refinement are contained in the SAINT+ (v6.45) and SHELXTL (v6.14) program suites, respectively (G. Sheldrick, Bruker AXS, Madison, WI).

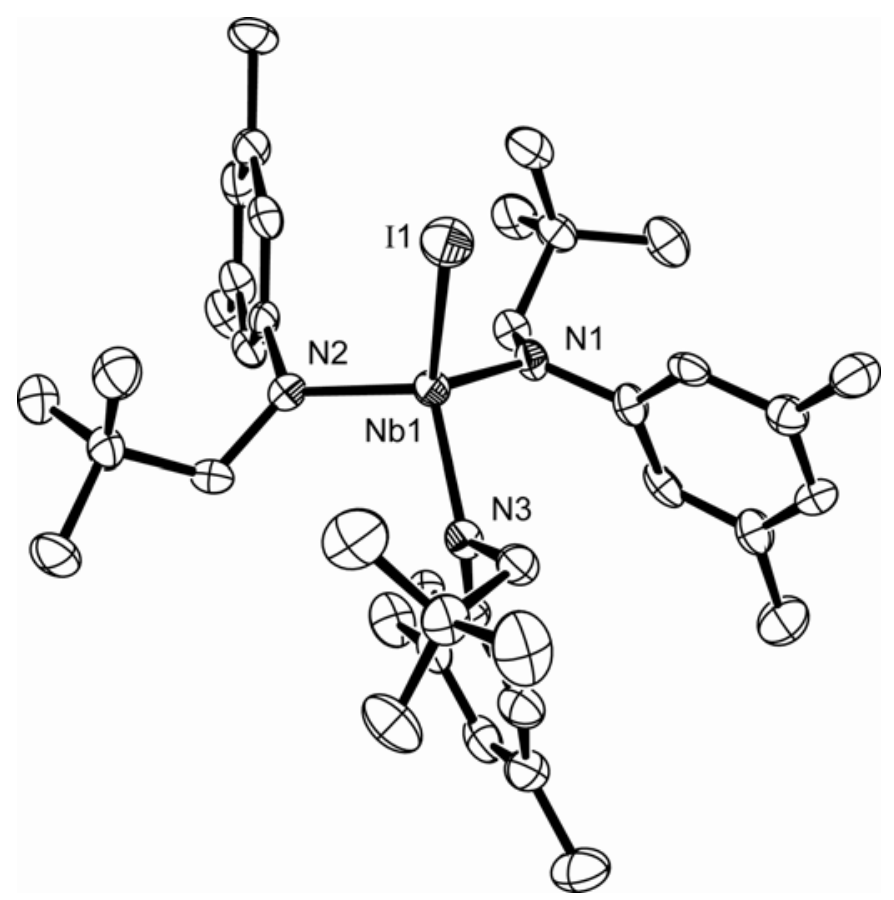

Figure S3.1. ORTEP Diagram of $\mathrm{Nb}(\mathrm{I})(\mathrm{N}[\mathrm{Np}] \mathrm{Ar})_{3}(\mathbf{1 a}-\mathrm{I})$ at the 35\% Probability Level. Selected Distances $(\AA)$ : Nb1-I1, 2.7294(9); Nb1-N1, 1.965(6); Nb1-N2, 1.980(6); Nb1-N3, 2.011(6). 


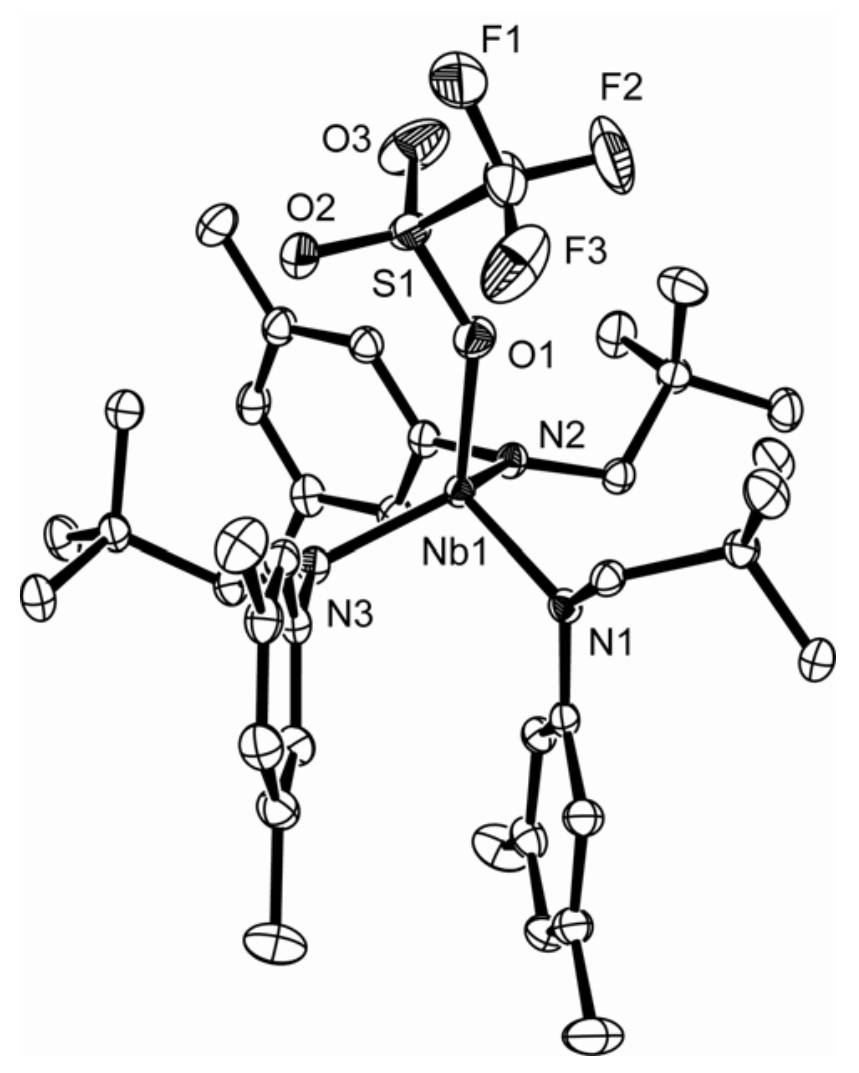

Figure S3.1. ORTEP Diagram of $\mathrm{Nb}(\mathrm{OTf})(\mathrm{N}[\mathrm{Np}] \mathrm{Ar})_{3}$ (1a-OTf) at the $50 \%$ Probability Level. Selected Distances ( $\AA$ ): Nb1O1, 2.0779(12); Nb1-N1, 1.9501(13); Nb1-N2, 1.9691(13); Nb1-N3, 2.0015(13).

Table S3.1. Crystallographic Data for Complexes $\mathbf{2 b}-\mathrm{H}, \mathrm{Na}(\mathrm{THF})_{3}[\mathbf{1 a}-\mathrm{N}], \mathbf{2 b}-\mathrm{N}$ and $\mathbf{1 c}-\mathrm{NC}(\mathrm{O}) t-\mathrm{Bu}$

\begin{tabular}{|c|c|c|c|c|}
\hline & 2b-H & $\mathrm{Na}(\mathrm{THF})_{3}[\mathbf{1 a}-\mathrm{N}]$ & 2b-N & 1c-NC(O)t-Bu \\
\hline Formula & $\mathrm{C}_{39} \mathrm{H}_{60} \mathrm{~N}_{3} \mathrm{Mo}$ & $\mathrm{C}_{51} \mathrm{H}_{84} \mathrm{~N}_{4} \overline{\mathrm{O}}_{3} \mathrm{NaNb}$ & $\overline{\mathrm{C}_{39}} \mathrm{H}_{60} \mathrm{~N}_{4} \overline{\mathrm{Mo}}$ & $\mathrm{C}_{41} \mathrm{H}_{63} \mathrm{~N}_{4} \mathrm{NbO}$ \\
\hline Crystal System & Monoclinic & Trigonal & Monoclinic & Monoclinic \\
\hline Space Group & $C_{2} / \mathrm{c}$ & $P-3$ & $P 2_{1} / \mathrm{c}$ & $P 2_{1} / \mathrm{n}$ \\
\hline$a, \AA$ & $38.035(3)$ & 15.8927(8) & $10.085(2)$ & $15.078(3)$ \\
\hline$b, \AA$ & 10.1353(9) & $15.8927(8)$ & $31.275(7)$ & $13.465(2)$ \\
\hline$c, \AA$ & 20.3079(18) & $14.4775(15)$ & 12.676(3) & 19.827(3) \\
\hline$\alpha, \operatorname{deg}$ & 90 & 90 & 90 & 90 \\
\hline$\beta$, deg & 103.9130(10) & 120 & $102.704(4)$ & $92.020(5)$ \\
\hline$\gamma, \operatorname{deg}$ & 90 & 90 & 90 & 90 \\
\hline$V, \AA^{3}$ & 7599.0(12) & $3166.8(4)$ & $3900.4(14)$ & $4022.9(11)$ \\
\hline $\mathrm{Z}$ & 8 & 2 & 4 & 4 \\
\hline radiation & & $\operatorname{Mo}-K_{\alpha}(\alpha=0.71073 \AA)$ & & \\
\hline $\mathrm{D}($ calcd $), \mathrm{g} / \mathrm{cm}^{3}$ & 1.166 & 0.962 & 1.159 & 1.190 \\
\hline$\mu(\mathrm{Mo} \mathrm{K} \alpha), \mathrm{mm}^{-1}$ & 0.373 & 0.231 & 0.366 & 0.333 \\
\hline temp, K & $183(2)$ & $183(2)$ & $193(2)$ & $100(2)$ \\
\hline No. Reflections & 14990 & 13348 & 20153 & 80576 \\
\hline No. Ind. Ref.( $\left.\mathrm{R}_{\text {int }}\right)$ & $5463(0.0644)$ & 3054(0.0299) & $6837(0.0378)$ & $9940(0.0611)$ \\
\hline $\mathrm{F}(000)$ & 2856 & 988 & 1456 & 1544 \\
\hline $\operatorname{GoF}\left(F^{2}\right)$ & 1.196 & 1.165 & 1.058 & 1.077 \\
\hline$R(F),{ }^{\mathrm{a}}$ & 0.0874 & 0.0606 & 0.0432 & 0.0898 \\
\hline$w R(F), \%^{\mathrm{a}}$ & 0.1739 & 0.1612 & 0.1144 & 0.2075 \\
\hline
\end{tabular}

${ }^{a}$ Quantity minimized $=w R\left(F^{2}\right)=\Sigma\left[w\left(F_{\mathrm{o}}{ }^{2}-{F_{\mathrm{c}}}^{2}\right)^{2}\right] / \Sigma\left[\left(w F_{\mathrm{o}}{ }^{2}\right)^{2}\right]^{1 / 2} ; R=\Sigma \Delta / \Sigma\left(F_{\mathrm{o}}\right), \Delta=\left|\left(F_{\mathrm{o}}-F_{\mathrm{c}}\right)\right|, w=1 /\left[\sigma 2\left(F_{\mathrm{o}}{ }^{2}\right)+(a P)^{2}+b P\right], P=\left[2 F_{\mathrm{c}}{ }^{2}+\right.$ $\left.\operatorname{Max}\left(F_{0}, 0\right)\right] / 3$. 
Table S3.2. Crystallographic Data for Complexes 1a-I and 1a-OTf

\begin{tabular}{|c|c|c|}
\hline & 1a-I & 1a-OTf \\
\hline Formula & $\mathrm{C}_{39} \mathrm{H}_{60} \mathrm{~N}_{3} \mathrm{NbI}$ & $\mathrm{C}_{40} \mathrm{H}_{60} \mathrm{~F}_{3} \mathrm{~N}_{3} \mathrm{NbO}_{3} \mathrm{~S}$ \\
\hline Crystal System & Monoclinic & Triclinic \\
\hline Space Group & $P 2_{1} / \mathrm{n}$ & $P-1$ \\
\hline$a, \AA$ & $12.5745(19)$ & $10.7040(5)$ \\
\hline$b, \AA$ & 19.634(3) & $11.6133(4)$ \\
\hline$c, \AA$ & $17.226(3)$ & 18.1727(8) \\
\hline$\alpha, \operatorname{deg}$ & 90 & $79.4720(10)$ \\
\hline$\beta$, deg & 109.859(2) & $75.2650(10)$ \\
\hline$\gamma$, deg & 90 & 75.9090(10) \\
\hline$V, \AA^{3}$ & $4000.0(10)$ & 2101.35(15) \\
\hline Z & 4 & 2 \\
\hline radiation & Mo-K ${ }_{\alpha}(\lambda$ & $3 \AA \AA)$ \\
\hline D(calcd), g/cm³ & 1.313 & 1.285 \\
\hline$\mu(\mathrm{Mo} \mathrm{K} \alpha), \mathrm{mm}^{-1}$ & 1.100 & 0.387 \\
\hline temp, K & 193(2) & $100(2)$ \\
\hline No. Reflections & 22967 & 43894 \\
\hline No. Ind. Ref.(R int $)$ & $6249(0.0408)$ & $10559(0.0325)$ \\
\hline $\mathrm{F}(000)$ & 1636 & 858 \\
\hline $\operatorname{GoF}\left(F^{2}\right)$ & 1.332 & 1.071 \\
\hline$R(F), \%^{\mathrm{a}}$ & 0.0808 & 0.0337 \\
\hline$w R(F), \%^{\mathrm{a}}$ & 0.1678 & 0.0884 \\
\hline
\end{tabular}

\section{References}

1. Ferris, T. D.;Lee, P. T.;Farrar, T. C. Mag. Res. Chem. 1997, 35, 571.

2. te Velde, G.; Bickelhaupt, F. M.; van Gisbergen, S. J. A.; Fonseca Guerra, C.; Baerends, E. J.; Snijders, J. G.; Ziegler, T. J. Comput. Chem. 2001, 22, 931.

3. Fonseca Guerra, C.; Snijders, J. G.; te Velde, G.; Baerends, E. J. Theor. Chem. Acc. 1998, 99, 391.

4. ADF 2004.01; SCM, Theoretical Chemistry, Vrije Universiteit, Amsterdam, The Netherlands, http://www.scm.com.

5. $\quad$ van Lenthe, E.; Baerends, E. J.; Snijders, J. G. J. Chem. Phys. 1993, 99, 4597.

6. van Lenthe, E. The ZORA Equation. Thesis, Vrije Universiteit Amsterdam, Netherlands, 1996.

7. Vosko, S. H.; Wilk, L.; Nusair, M. Can. J. Phys. 1980, 58, 1200-1211.

8. Becke, A. Phys. Rev.. A 1988, 38, 3098-3100.

9. Perdew, J. P. Phys. Rev. B 1986, 34, 7406-7406.

10. Perdew, J. P. Phys. Rev. B 1986, 33, 8822-8824.

11. See, for example: Deng, L.; Schmid, R.; Ziegler, T. Organometallics 2000, 19, 3069-3076 